Research Article

\title{
A Group Theory Approach towards Some Rational Difference Equations
}

\author{
Mensah Folly-Gbetoula (D), Nkosingiphile Mnguni, and A. H. Kara \\ School of Mathematics, University of the Witwatersrand, Private Bag 3, Johannesburg 2050, South Africa \\ Correspondence should be addressed to Mensah Folly-Gbetoula; mensah.folly-gbetoula@wits.ac.za
}

Received 5 September 2019; Accepted 8 November 2019; Published 1 December 2019

Academic Editor: Francisco Balibrea

Copyright (c) 2019 Mensah Folly-Gbetoula et al. This is an open access article distributed under the Creative Commons Attribution License, which permits unrestricted use, distribution, and reproduction in any medium, provided the original work is properly cited.

A full Lie point symmetry analysis of rational difference equations is performed. Nontrivial symmetries are derived, and exact solutions using these symmetries are obtained.

\section{Introduction}

Over a century ago, symmetries became a centre of interest of several authors after the work of Sophus Lie [1] on differential equations. He studied the continuous group of transformations that leaves the differential equations invariant. This concept of symmetries is strongly related to the existence of conservation laws, and the relationship between them has attracted great interest among researchers following the work of Noether [2]. The extension of this idea to difference equations is now well documented (see [3] and references herein). In [3], Hydon developed a symmetrybased algorithm enabling one to derive solutions of difference equations without making any special lucky guesses. Hydon emphasized on second-order difference equations, although his algorithm is valid for any order. When it comes to higher-order equations, computations can be cumbersome and extra ansatz may be needed to ease the calculations.

We aim to extend the work by Elsayed [4] where the author investigated the dynamics and solutions of

$$
x_{n+1}=\frac{x_{n-5}}{ \pm 1 \pm x_{n-1} x_{n-3} x_{n-5}},
$$

where the initial conditions $x_{-5}, x_{-4}, x_{-3}, x_{-2}, x_{-1}$, and $x_{0}$ are arbitrary nonzero real numbers. For related work, see $[5,6]$. One can notice that equation (1) is just a special case of a more general form

$$
x_{n+1}=\frac{x_{n-5}}{a_{n}+b_{n} x_{n-1} x_{n-3} x_{n-5}}
$$

where $\left(a_{n}\right)_{n \in \mathbb{N}_{0}}$ and $\left(b_{n}\right)_{n \in \mathbb{N}_{0}}$ are arbitrary sequences. We will use a symmetry-based method to solve (2). Equivalently, we study

$$
u_{n+6}=\frac{u_{n}}{A_{n}+B_{n} u_{n} u_{n+2} u_{n+4}}
$$

instead, where $\left(A_{n}\right)_{n \in \mathbb{N}_{0}}$ and $\left(B_{n}\right)_{n \in \mathbb{N}_{0}}$ are arbitrary sequences. This means we can only compare $x_{i}$ with $u_{i+5}$. Furthermore, we use the same technique to obtain exact solutions of

$$
x_{n+1}=\frac{x_{n-4} x_{n-3} x_{n-2}}{x_{n-1} x_{n}\left(\lambda+\mu x_{n-4} x_{n-3} x_{n-2}\right)},
$$

where $\lambda, \mu \in \mathbb{R}$, and again we study

$$
u_{n+5}=\frac{u_{n} u_{n+1} u_{n+2}}{u_{n+3} u_{n+4}\left(\lambda+\mu u_{n} u_{n+1} u_{n+2}\right)}
$$

instead. Note that solutions of (4) are found in [7]; however, their method is completely different from ours.

\section{Definitions and Notation}

The definitions are taken from Hydon [3], and most of the notation follows from the same book. 
Definition 1. A parameterized set of point transformations,

$$
\Gamma_{\varepsilon}: x \longmapsto \hat{x}(x ; \varepsilon),
$$

where $x=x_{i}$ and $i=1, \ldots, p$ are continuous variables, is a one-parameter local Lie group of transformations if the following conditions are satisfied:

(1) $\Gamma_{0}$ is the identity map if $\hat{x}=x$ when $\varepsilon=0$

(2) $\Gamma_{a} \Gamma_{b}=\Gamma_{a+b}$ for every $a$ and $b$ sufficiently close to 0

(3) Each $\hat{x}_{i}$ can be represented as a Taylor series (in a neighborhood of $\varepsilon=0$ that is determined by $x$ ), and therefore

$$
\widehat{x}_{i}(x ; \varepsilon)=x_{i}+\varepsilon \xi_{i}(x)+O\left(\varepsilon^{2}\right), \quad i=1, \ldots, p .
$$

Consider the $p$ th-order difference equation

$$
u_{n+p}=\Omega\left(n, u_{n}, \ldots, u_{n+p-1}\right)
$$

for some function $\Omega$. Assume the point transformations are of the form

$$
\begin{aligned}
\widehat{n} & =n, \\
\widehat{u}_{n} & =u_{n}+\varepsilon Q\left(n, u_{n}\right)+O\left(\varepsilon^{2}\right),
\end{aligned}
$$

with the corresponding infinitesimal symmetry generator

$$
\begin{aligned}
X= & Q\left(n, u_{n}\right) \frac{\partial}{\partial u_{n}}+S Q\left(n, u_{n}\right) \frac{\partial}{\partial u_{n+1}}+\cdots \\
& +S^{(p-1)} Q\left(n, u_{n}\right) \frac{\partial}{\partial u_{n+p-1}},
\end{aligned}
$$

where $S$ is the shift operator, i.e., $S: n \longmapsto n+1$. The symmetry condition is defined as

$$
\widehat{u}_{n+p}=\Omega\left(n, \widehat{u}_{n}, \widehat{u}_{n+1}, \ldots, \widehat{u}_{n+p-1}\right),
$$

whenever (8) is true. Substituting the Lie point symmetries (9) into the symmetry condition (11) leads to the linearized symmetry condition:

$$
S^{(p)} Q-X \Omega=0,
$$

whenever (8) holds.

Definition 2. $V_{n}$ is invariant under the Lie group of transformations (9) if and only if $X V_{n}=0$.

We define the functions

$$
\Theta^{n}\left(\theta_{j}, s, l\right)=\prod_{j=s}^{l} \theta_{2 j+n}, \quad n=0,1,
$$

and we adopt the standard conventions

$$
\begin{aligned}
& \prod_{j=s}^{l} \theta_{j}=1, \quad \text { when } s>l, \\
& \sum_{j=n}^{l_{0}} \theta_{j}=0, \quad \text { when } n>l_{0} .
\end{aligned}
$$

We refer the reader to [3] for a deeper understanding of the concept of symmetry analysis of difference equations.

\section{Main Results}

3.1. On the Difference Equation (3). Consider the sixth-order difference equations of the form (3), that is,

$$
u_{n+6}=\frac{u_{n}}{A_{n}+B_{n} u_{n} u_{n+2} u_{n+4}} .
$$

We impose the symmetry condition (12) and simplify the resulting equation to get

$$
S^{6} Q+\frac{B_{n} u_{n}^{2} u_{n+2} S^{4} Q+B_{n} u_{n}^{2} u_{n+4} S^{2} Q-A_{n} Q}{\left(B_{n} u_{n} u_{n+2} u_{n+4}+A_{n}\right)^{2}}=0 .
$$

To solve for $Q$, we first differentiate (16) with respect to $u_{n}$ (keeping $\Omega$ fixed and viewing $u_{n+2}$ as a function of $u_{n}, u_{n+4}$, and $\Omega$ ). This leads, after simplification, to

$$
S^{4} Q+u_{n+4}\left(S^{2} Q\right)^{\prime}-u_{n+4} Q^{\prime}+\frac{2 u_{n+4}}{u_{n}} Q=0,
$$

where ' denotes the derivative with respect to the independent variable. We then differentiate (17) with respect to $u_{n}$ to get

$$
Q^{\prime \prime}\left(n, u_{n}\right)-\frac{2}{u_{n}} Q^{\prime}\left(n, u_{n}\right)+\frac{2}{u_{n}^{2}} Q\left(n, u_{n}\right)=0 .
$$

The solutions of (18) are given by

$$
Q\left(n, u_{n}\right)=\alpha_{n} u_{n}^{2}+\beta_{n} u_{n}
$$

for some functions $\alpha_{n}$ and $\beta_{n}$ of $n$. To obtain more information on $\alpha_{n}$ and $\beta_{n}$, we substitute (19) in (16) and we split the resulting equation to get the following:

$$
\begin{aligned}
& u_{n} u_{n+2} u_{n+4}^{2}: B_{n} \alpha_{n+4}=0, \\
& u_{n} u_{n+2}^{2} u_{n+4}: B_{n} \alpha_{n+2}=0, \\
& u_{n} u_{n+2} u_{n+4}: B_{n}\left(\beta_{n+2}+\beta_{n+4}+\beta_{n+6}\right)=0,
\end{aligned}
$$

$$
\begin{gathered}
u_{n}:-A_{n} \alpha_{n}+\alpha_{n+6}=0, \\
1: A_{n}\left(\beta_{n}-\beta_{n+6}\right)=0 .
\end{gathered}
$$

These equations $((20 \mathrm{a})-(20 \mathrm{e}))$ reduce to

$$
\begin{aligned}
\alpha_{n} & =0, \\
\beta_{n+4}+\beta_{n+2}+\beta_{n} & =0 .
\end{aligned}
$$

The expression of $\beta_{n}$ in (21) is merely obtained by solving the corresponding characteristic equation $r^{4}+r^{2}+1=0$ for $r$. Assuming that $r_{i}, i=1,2,3,4$, are the solutions of this characteristic equation, then $\beta_{n}$ is a linear combination of the $r_{i}^{n}$ 's. In other words, the solutions of (21) are

$$
\begin{aligned}
& \alpha_{n}=0, \\
& \beta_{n}=(-1)^{n} \beta^{n} c_{1}+(-1)^{n} \bar{\beta}^{n} c_{2}+\bar{\beta}^{n} c_{3}+\beta^{n} c_{4},
\end{aligned}
$$


for some arbitrary constants $c_{i}, i=1, \ldots, 4$, where $\beta=\exp (\pi i / 3)$. Thus, we obtain four characteristics given by

$$
\begin{aligned}
& Q_{1}=(-1)^{n} \beta^{n} u_{n}, \\
& Q_{2}=(-1)^{n} \bar{\beta}^{n} u_{n}, \\
& Q_{3}=\bar{\beta}^{n} u_{n}, \\
& Q_{4}=\beta^{n} u_{n} .
\end{aligned}
$$

The four corresponding symmetry generators $X_{1}, X_{2}, X_{3}$, and $X_{4}$ are given by

$$
\begin{aligned}
X_{1}= & (-1)^{n} \beta^{n} u_{n} \partial u_{n}-(-1)^{n} \beta^{n+1} u_{n+1} \partial_{u_{n+1}}+(-1)^{n} \beta^{n+2} u_{n+2} \partial_{u_{n+2}} \\
& -(-1)^{n} \beta^{n+3} u_{n+3} \partial_{u_{n+3}}+(-1)^{n} \beta^{n+4} u_{n+4} \partial_{u_{n+4}}-(-1)^{n} \beta^{n+5} u_{n+5} \partial_{u_{n+5}}, \\
X_{2}= & (-1)^{n} \bar{\beta}^{n} u_{n} \partial u_{n}-(-1)^{n} \bar{\beta}^{n+1} u_{n+1} \partial_{u_{n+1}}+(-1)^{n} \bar{\beta}^{n+2} u_{n+2} \partial_{u_{n+2}} \\
& -(-1)^{n} \bar{\beta}^{n+3} u_{n+3} \partial_{u_{n+3}}+(-1)^{n} \bar{\beta}^{n+4} u_{n+4} \partial_{u_{n+4}}-(-1)^{n} \beta^{n+5} u_{n+5} \partial_{u_{n+5}}, \\
X_{3}= & \bar{\beta}^{n} u_{n} \partial u_{n}+\bar{\beta}^{n+1} u_{n+1} \partial_{u_{n+1}}+\bar{\beta}^{n+2} u_{n+2} \partial_{u_{n+2}}+\bar{\beta}^{n+3} u_{n+3} \partial_{u_{n+3}} \\
& +\bar{\beta}^{n+4} u_{n+4} \partial_{u_{n+4}}+\bar{\beta}^{n+5} u_{n+5} \partial_{u_{n+5}} \\
X_{4}= & \beta^{n} u_{n} \partial u_{n}+\beta^{n+1} u_{n+1} \partial_{u_{n+1}}+\beta^{n+2} u_{n+2} \partial_{u_{n+2}}+\beta^{n+3} u_{n+3} \partial_{u_{n+3}} \\
& +\beta^{n+4} u_{n+4} \partial_{u_{n+4}}+\beta^{n+5} u_{n+5} \partial_{u_{n+5} .}
\end{aligned}
$$

Here, using $Q_{4}$, we introduce the canonical coordinate [8]

$$
s_{n}=\int \frac{d u_{n}}{\beta^{n} u_{n}}=\frac{1}{\beta^{n}} \ln \left|u_{n}\right| .
$$

In view of (21), we introduce the variable

$$
r_{n}=\beta^{n+4} s_{n+4}+\beta^{n+2} s_{n+2}+\beta^{n} s_{n} .
$$

It is easy to check that

$$
X_{i} r_{n}=\beta^{n+4}+\beta^{n+2}+\beta^{n}=0, \quad i=1, \ldots, 4 .
$$

Therefore, $r_{n}$ is invariant under $X_{4}$. It is advantageous to use

$$
\left|\widetilde{r}_{n}\right|=\exp \left(-r_{n}\right) \text {, }
$$

that is, $\widetilde{r}_{n}= \pm 1 /\left(u_{n} u_{n+2} u_{n+4}\right)$. Here, we choose to use the plus sign and we show, using (3), that

$$
\tilde{r}_{n+2}=A_{n} \tilde{r}_{n}+B_{n} \text {. }
$$

Therefore,

$$
\widetilde{r}_{2 n+k}=\widetilde{r}_{k}\left(\prod_{k_{1}=0}^{n-1} A_{2 k_{1}+k}\right)+\sum_{l=0}^{n-1}\left(B_{2 l+k} \prod_{k_{2}=l+1}^{n-1} A_{2 k_{2}+k}\right), \quad k=0,1 .
$$

It is worthwhile to mention that equation (30) gives the solution of (29) for all $n$. By reversing all the change of variables, we have

$$
\begin{aligned}
\left|u_{n}\right|= & \exp \left[(-\beta)^{n} c_{5}+(\beta)^{n} c_{6}+(\bar{\beta})^{n} c_{7}+(-\bar{\beta})^{n} c_{8}+(-\beta)^{n+1} \sum_{k_{1}=0}^{n-1} \frac{-i \sqrt{3}}{6}(-\bar{\beta})^{k_{1}} r_{k_{1}}+(\beta)^{n+1} \sum_{k_{2}=0}^{n-1} \frac{i \sqrt{3}}{6}(\bar{\beta})^{k_{2}} r_{k_{2}}\right. \\
& \left.+(\bar{\beta})^{n+1} \sum_{k_{3}=0}^{n-1} \frac{-i \sqrt{3}}{6} \beta^{k_{3}} r_{k_{3}}+(-\bar{\beta})^{n+1} \sum_{k_{4}=0}^{n-1} \frac{i \sqrt{3}}{6}(-\beta)^{k_{4}} r_{k_{4}}\right] \\
= & \Gamma_{n} \exp \left(\sum_{k=0}^{n-1} \frac{\sqrt{3}}{3}\left[(-1)^{n+k}+1\right] \operatorname{Im}[\gamma(n+1, k)] \ln \left|\widetilde{r}_{k}\right|\right),
\end{aligned}
$$


where $\tilde{r}_{k}$ is given in (30), $\quad \Gamma_{n}=\exp \left\{(-\beta)^{n} c_{5}+\right.$ $\left.\beta^{n} c_{6}+\bar{\beta}^{n} c_{7}+(-\bar{\beta})^{n} c_{8}\right\}$, and $\gamma(l, k)=\beta^{l} \bar{\beta}^{k}$.

Note. Equation (31) gives the solution of (3) in a unified manner.

We can simplify (31) further by splitting it into six categories. We have

$$
\begin{aligned}
\left|u_{6 n}\right| & =\Gamma_{6 n} \exp \left(\sum_{k=0}^{6 n-1} \frac{\sqrt{3}}{3}\left[(-1)^{6 n+k}+1\right] \operatorname{Im}[\gamma(6 n+1, k)] \ln \left|\widetilde{r}_{k}\right|\right), \\
u_{6 n} & =u_{0} \prod_{s=0}^{n-1} \frac{\widetilde{r}_{6 s}}{\widetilde{r}_{6 s+2}} .
\end{aligned}
$$

Similarly, after a straightforward but lengthy computation, we get

$$
u_{6 n+i}=u_{i} \prod_{s=0}^{n-1} \frac{\widetilde{r}_{6 s+i}}{\widetilde{r}_{6 s+2+i}}, \quad i=0, \ldots, 5 .
$$

Note. We can obtain (33) using (28) without considering the absolute value function.

Now, using (30) and (33), we obtain the solutions of (3) as follows:

$$
\begin{gathered}
u_{6 n}=u_{0} \prod_{s=0}^{n-1} \frac{\left(\prod_{k_{1}=0}^{3 s-1} A_{2 k_{1}}\right)+u_{0} u_{2} u_{4} \sum_{l=0}^{3 s-1}\left(B_{2 l} \prod_{k_{2}=l+1}^{3 s-1} A_{2 k_{2}}\right)}{\left(\prod_{k_{1}=0}^{3 s} A_{2 k_{1}}\right)+u_{0} u_{2} u_{4} \sum_{l=0}^{3 s}\left(B_{2 l} \prod_{k_{2}=l+1}^{3 s} A_{2 k_{2}}\right)}, \\
u_{6 n+1}=u_{1} \frac{\left(\prod_{1_{1}=0}^{3 s-1} A_{2 k_{1}+1}\right)+u_{1} u_{3} u_{5} \sum_{l=0}^{3 s-1}\left(B_{2 l+1} \prod_{k_{2}=l+1}^{3 s-1} A_{2 k_{2}+1}\right)}{\left(\prod_{k_{1}=0}^{3 s} A_{2 k_{1}+1}\right)+u_{1} u_{3} u_{5} \sum_{l=0}^{3 s}\left(B_{2 l+1} \prod_{k_{2}=l+1}^{3 s} A_{2 k_{2}+1}\right)}, \\
u_{6 n+2}=u_{2} \prod_{s=0}^{n-1} \frac{\left(\prod_{k_{1}=0}^{3 s} A_{2 k_{1}}\right)+u_{0} u_{2} u_{4} \sum_{l=0}^{3 s}\left(B_{2 l} \prod_{k_{2}=l+1}^{3 s} A_{2 k_{2}}\right)}{\left(\prod_{k_{1}=0}^{3 s+1} A_{2 k_{1}}\right)+u_{0} u_{2} u_{4} \sum_{l=0}^{3 s+1}\left(B_{2 l} \prod_{k_{2}=l+1}^{3 s+1} A_{2 k_{2}}\right)}, \\
u_{6 n+3}=u_{3} \prod_{s=0}^{n-1} \frac{\left(\prod_{k_{1}=0}^{3 s} A_{2 k_{1}+1}\right)+u_{1} u_{3} u_{5} \sum_{l=0}^{3 s}\left(B_{2 l+1} \prod_{k_{2}=l+1}^{3 s} A_{2 k_{2}+1}\right)}{\left(\prod_{k_{1}=0}^{3 s+1} A_{2 k_{1}+1}\right)+u_{1} u_{3} u_{5} \sum_{l=0}^{3 s+1}\left(B_{2 l+1} \prod_{k_{2}=l+1}^{3 s+1} A_{2 k_{2}+1}\right)}, \\
u_{6 n+4}=u_{4} \prod_{s=0}^{n-1} \frac{\left(\prod_{k_{1}=0}^{3 s+1} A_{2 k_{1}}\right)+u_{0} u_{2} u_{4} \sum_{l=0}^{3 s+1}\left(B_{2 l} \prod_{k_{2}=l+1}^{3 s+1} A_{2 k_{2}}\right)}{\left(\prod_{k_{1}=0}^{3 s+2} A_{2 k_{1}}\right)+u_{0} u_{2} u_{4} \sum_{l=0}^{3 s+2}\left(B_{2 l} \prod_{k_{2}=l+1}^{3 s+2} A_{2 k_{2}}\right)}, \\
u_{6 n+5}=u_{5} \prod_{s=0}^{n-1} \frac{\left(\prod_{k_{1}=0}^{3 s+1} A_{2 k_{1}+1}\right)+u_{1} u_{3} u_{5} \sum_{l=0}^{3 s+1}\left(B_{2 l+1} \prod_{k_{2}=l+1}^{3 s+1} A_{2 k_{2}+1}\right)}{\left(\prod_{k_{1}=0}^{3 s+2} A_{2 k_{1}+1}\right)+u_{1} u_{3} u_{5} \sum_{l=0}^{3 s+2}\left(B_{2 l+1} \prod_{k_{2}=l+1}^{3 s+2} A_{2 k_{2}+1}\right)},
\end{gathered}
$$

whenever the denominators do not vanish, i.e., The implication is that solutions of (2) are given by $u_{0} u_{2} u_{4} \sum_{l=0}^{s} B_{2 l} \Theta^{0}\left(A_{k_{2}}, l+1, s\right) \neq-\Theta^{0}\left(A_{k_{1}}, 0, s\right)$ and $u_{1} u_{3} u_{5} \sum_{l=0}^{s} B_{2 l+1} \Theta^{1}\left(A_{k_{2}}, l+1, s\right) \neq-\Theta^{1}\left(A_{k_{1}}, 0, s\right)$.

$$
\begin{aligned}
& x_{6 n-5}=x_{-5} \prod_{s=0}^{n-1} \frac{\left(\prod_{k_{1}=0}^{3 s-1} a_{2 k_{1}}\right)+x_{-5} x_{-3} x_{-1} \sum_{l=0}^{3 s-1}\left(b_{2 l} \prod_{k_{2}=l+1}^{3 s-1} a_{2 k_{2}}\right)}{\left(\prod_{k_{1}=0}^{3 s} a_{2 k_{1}}\right)+x_{-5} x_{-3} x_{-1} \sum_{l=0}^{3 s}\left(b_{2 l} \prod_{k_{2}=l+1}^{3 s} a_{2 k_{2}}\right)}, \\
& x_{6 n-4}=x_{-4} \prod_{s=0}^{n-1} \frac{\left(\prod_{k_{1}=0}^{3 s-1} a_{2 k_{1}+1}\right)+x_{-4} x_{-2} x_{0} \sum_{l=0}^{3 s-1}\left(b_{2 l+1} \prod_{k_{2}=l+1}^{3 s-1} a_{2 k_{2}+1}\right)}{\left(\prod_{k_{1}=0}^{3 s} a_{2 k_{1}+1}\right)+x_{-4} x_{-2} x_{0} \sum_{l=0}^{3 s}\left(b_{2 l+1} \prod_{k_{2}=l+1}^{3 s} a_{2 k_{2}+1}\right)}, \\
& x_{6 n-3}=x_{-3} \prod_{s=0}^{n-1} \frac{\left(\prod_{k_{1}=0}^{3 s} a_{2 k_{1}}\right)+x_{-5} x_{-3} x_{-1} \sum_{l=0}^{3 s}\left(b_{2 l} \prod_{k_{2}=l+1}^{3 s} a_{2 k_{2}}\right)}{\left(\prod_{k_{1}=0}^{3 s+1} a_{2 k_{1}}\right)+x_{-5} x_{-3} x_{-1} \sum_{l=0}^{3 s+1}\left(b_{2 l} \prod_{k_{2}=l+1}^{3 s+1} a_{2 k_{2}}\right)},
\end{aligned}
$$




$$
\begin{gathered}
x_{6 n-2}=x_{-2} \prod_{s=0}^{n-1} \frac{\left(\prod_{k_{1}=0}^{3 s} a_{2 k_{1}+1}\right)+x_{-4} x_{-2} x_{0} \sum_{l=0}^{3 s}\left(b_{2 l+1} \prod_{k_{2}=l+1}^{3 s} a_{2 k_{2}+1}\right)}{\left(\prod_{k_{1}=0}^{3 s+1} a_{2 k_{1}+1}\right)+x_{-4} x_{-2} x_{0} \sum_{l=0}^{3 s+1}\left(b_{2 l+1} \prod_{k_{2}=l+1}^{3 s+1} a_{2 k_{2}+1}\right)}, \\
x_{6 n-1}=x_{-1} \prod_{s=0}^{n-1} \frac{\left(\prod_{k_{1}=0}^{3 s+1} a_{2 k_{1}}\right)+x_{-5} x_{-3} x_{-1} \sum_{l=0}^{3 s+1}\left(b_{2 l} \prod_{k_{2}=l+1}^{3 s+1} a_{2 k_{2}}\right)}{\left(\prod_{k_{1}=0}^{3 s+2} a_{2 k_{1}}\right)+x_{-5} x_{-3} x_{-1} \sum_{l=0}^{3 s+2}\left(b_{2 l} \prod_{k_{2}=l+1}^{3 s+2} a_{2 k_{2}}\right)}, \\
x_{6 n}=x_{0} \prod_{s=0}^{n-1} \frac{\left(\prod_{k_{1}=0}^{3 s+1} a_{2 k_{1}+1}\right)+x_{-4} x_{-2} x_{0} \sum_{l=0}^{3 s+1}\left(b_{2 l+1} \prod_{k_{2}=l+1}^{3 s+1} a_{2 k_{2}+1}\right)}{\left(\prod_{k_{1}=0}^{3 s+2} a_{2 k_{1}+1}\right)+x_{-4} x_{-2} x_{0} \sum_{l=0}^{3 s+2}\left(b_{2 l+1} \prod_{k_{2}=l+1}^{3 s+2} a_{2 k_{2}+1}\right)},
\end{gathered}
$$

whenever the denominators do not vanish, i.e., $x_{-5} x_{-3} x_{-1} \sum_{l=0}^{s} b_{2 l} \Theta^{0}\left(a_{k_{2}}, l+1, s\right) \neq-\Theta^{0}\left(a_{k_{1}}, 0, s\right)$ and $x_{-4} x_{-2}$ $x_{-0} \sum_{l=0}^{s} b_{2 l+1} \Theta^{1}\left(a_{k_{2}}, l+1, s\right) \neq-\Theta^{1}\left(a_{k_{1}}, 0, s\right)$.

3.2. The Case Where $\left(a_{n}\right)$ and $\left(b_{n}\right)$ Are Two Periodic Sequences. Let $a_{n}=(\lambda, \mu, \lambda, \mu, \lambda, \ldots), b_{n}=(\eta, \zeta, \eta, \zeta, \ldots), \Phi=x_{-5} x_{-3} x_{-1}$, and $\Psi=x_{-4} x_{-2} x_{0}$. The solution in this case is given by the following equations:

$$
\begin{aligned}
& x_{6 n-5}=x_{-5} \prod_{s=0}^{n-1} \frac{\lambda^{3 s}+\eta \Phi \sum_{l=0}^{3 s-1} \lambda^{l}}{\lambda^{3 s+1}+\eta \Phi \sum_{l=0}^{3 s} \lambda^{l}}, \\
& x_{6 n-4}=x_{-4} \prod_{s=0}^{n-1} \frac{\mu^{3 s}+\zeta \Psi \sum_{l=0}^{3 s-1} \mu^{l}}{\mu^{3 s+1}+\zeta \Psi \sum_{l=0}^{3 s} \mu^{l}}, \\
& x_{6 n-3}=x_{-3} \prod_{s=0}^{n-1} \frac{\lambda^{3 s+1}+\eta \Phi \sum_{l=0}^{3 s} \lambda^{l}}{\lambda^{3 s+2}+\eta \Phi \sum_{l=0}^{3 s+1} \lambda^{l}}, \\
& x_{6 n-2}=x_{-2} \prod_{s=0}^{n-1} \frac{\mu^{3 s+1}+\zeta \Psi \sum_{l=0}^{3 s} \mu^{l}}{\mu^{3 s+2}+\zeta \Psi \sum_{l=0}^{3 s+1} \mu^{l}}, \\
& x_{6 n-1}=x_{-1} \prod_{s=0}^{n-1} \frac{\lambda^{3 s+2}+\eta \Phi \sum_{l=0}^{3 s+1} \lambda^{l}}{\lambda^{3 s+3}+\eta \Phi \sum_{l=0}^{3 s+2} \lambda^{l}}, \\
& x_{6 n}=x_{0} \prod_{s=0}^{n-1} \frac{\mu^{3 s+2}+\zeta \Psi \sum_{l=0}^{3 s+1} \mu^{l}}{\mu^{3 s+3}+\zeta \Psi \sum_{l=0}^{3 s+2} \mu^{l}},
\end{aligned}
$$

where $\left(\sum_{l=0}^{s-1} \lambda^{l}\right) \eta \Phi \neq-\lambda^{s}$ and $\left(\sum_{l=0}^{s-1} \lambda^{l}\right) \eta \Psi \neq-\lambda^{s}, s \leq 3 n$.

3.3. The Case Where $\left(a_{n}\right)$ and $\left(b_{n}\right)$ Are Constants. Here, $a_{n}=\mu=\lambda, b_{n}=\eta=\zeta, \Phi=x_{-5} x_{-3} x_{-1}$, and $\Psi=x_{-4} x_{-2} x_{0}$. Equation (2) becomes $x_{n+1}=x_{n-5} /\left(\lambda+\eta x_{n-1} x_{n-3} x_{n-5}\right)$.

3.3.1. $\lambda=1$. The solution given in (35a)-(35f) simplifies to

$$
\begin{aligned}
& x_{6 n-5}=x_{-5} \prod_{s=0}^{n-1} \frac{1+(3 s) \eta \Phi}{1+(3 s+1) \eta \Phi} \\
& x_{6 n-4}=x_{-4} \prod_{s=0}^{n-1} \frac{1+(3 s) \eta \Psi}{1+(3 s+1) \eta \Psi} \\
& x_{6 n-3}=x_{-3} \prod_{s=0}^{n-1} \frac{1+(3 s+1) \eta \Phi}{1+(3 s+2) \eta \Phi} \\
& x_{6 n-2}=x_{-2} \prod_{s=0}^{n-1} \frac{1+(3 s+1) \eta \Psi}{1+(3 s+2) \eta \Psi} \\
& x_{6 n-1}=x_{-1} \prod_{s=0}^{n-1} \frac{1+(3 s+2) \eta \Phi}{1+(3 s+3) \eta \Phi} \\
& x_{6 n}=x_{0} \prod_{s=0}^{n-1} \frac{1+(3 s+2) \eta \Psi}{1+(3 s+3) \eta \Psi}
\end{aligned}
$$

where $s \eta \Phi \neq-1$ and $s \eta \Psi \neq-1, s \leq 3 n$.

(i) If $\eta=1$, then equations in (37a)-(37c) are exactly the ones obtained in Theorem 2.1 in [4] for

$$
x_{n+1}=\frac{x_{n-5}}{1+x_{n-1} x_{n-3} x_{n-5}}
$$

and their restriction (the initial conditions are arbitrary nonzero positive real numbers) is a special case of our restriction (the initial conditions are arbitrary nonzero real numbers and $s \Phi \neq-1, s \Psi \neq-1$, and $0 \leq s \leq 3 n)$.

(ii) If $\eta=-1$, then equations in (37a)-(37c) are exactly the ones obtained in Theorem 3.1 in [4] for

$$
x_{n+1}=\frac{x_{n-5}}{1-x_{n-1} x_{n-3} x_{n-5}}
$$

and their restriction (the initial conditions are arbitrary nonzero real numbers and $j b d f \neq 1$ and jace $\neq 1$ ) coincides with our restriction (the initial conditions are arbitrary nonzero real numbers and $s \Phi \neq 1, s \Psi \neq 1$, and $0 \leq s \leq 3 n)$. 
3.3.2. The Case Where $\lambda \neq 1$. In this case, the solution given in (35a)-(35f) simplifies to

$$
\begin{aligned}
& x_{6 n-5}=x_{-5} \prod_{s=0}^{n-1} \frac{\lambda^{3 s}+\eta \Phi\left(\left(1-\lambda^{3 s}\right) /(1-\lambda)\right)}{\lambda^{3 s+1}+\eta \Phi\left(\left(1-\lambda^{3 s+1}\right) /(1-\lambda)\right)} \\
& x_{6 n-4}=x_{-4} \prod_{s=0}^{n-1} \frac{\lambda^{3 s}+\zeta \Psi\left(\left(1-\lambda^{3 s}\right) /(1-\lambda)\right)}{\lambda^{3 s+1}+\zeta \Psi\left(\left(1-\lambda^{3 s+1}\right) /(1-\lambda)\right)} \\
& x_{6 n-3}=x_{-3} \prod_{s=0}^{n-1} \frac{\lambda^{3 s+1}+\eta \Phi\left(\left(1-\lambda^{3 s+1}\right) /(1-\lambda)\right)}{\lambda^{3 s+2}+\eta \Phi\left(\left(1-\lambda^{3 s+2}\right) /(1-\lambda)\right)} \\
& x_{6 n-2}=x_{-2} \prod_{s=0}^{n-1} \frac{\lambda^{3 s+1}+\zeta \Psi\left(\left(1-\lambda^{3 s+1}\right) /(1-\lambda)\right)}{\lambda^{3 s+2}+\zeta \Psi\left(\left(1-\lambda^{3 s+2}\right) /(1-\lambda)\right)} \\
& x_{6 n-1}=x_{-1} \prod_{s=0}^{n-1} \frac{\lambda^{3 s+2}+\eta \Phi\left(\left(1-\lambda^{3 s+2}\right) /(1-\lambda)\right)}{\lambda^{3 s+3}+\eta \Phi\left(\left(1-\lambda^{3 s+3}\right) /(1-\lambda)\right)} \\
& x_{6 n}=x_{0} \prod_{s=0}^{n-1} \frac{\lambda^{3 s+2}+\zeta \Psi\left(\left(1-\lambda^{3 s+2}\right) /(1-\lambda)\right)}{\lambda^{3 s+3}+\zeta \Psi\left(\left(1-\lambda^{3 s+3}\right) /(1-\lambda)\right)}
\end{aligned}
$$

where $\left(1-\lambda^{s}\right) \eta \Phi \neq-\lambda^{s}(1-\lambda)$ and $\left(1-\lambda^{s}\right) \zeta \Psi \neq-\lambda^{s}(1-$ $\lambda), s \leq 3 n$.

Note. If $\lambda=-1$, then the solution given in Section 3.3.2 simplifies to

$$
\begin{aligned}
x_{12 n-5} & =x_{6(2 n)-5}=x_{-5}, \\
x_{12 n-4} & =x_{-4}, \\
x_{12 n-3} & =x_{-3}, \\
x_{12 n-2} & =x_{-2}, \\
x_{12 n-1} & =x_{-1}, \\
x_{12 n} & =x_{0}, \\
x_{12 n+1} & =\frac{x_{-5}}{-1+\eta \Phi}, \\
x_{12 n+2} & =\frac{x_{-4}}{-1+\zeta \Psi}, \\
x_{12 n+3} & =x_{-3}(-1+\eta \Phi), \\
x_{12 n+4} & =x_{-2}(-1+\zeta \Psi), \\
x_{12 n+5} & =\frac{x_{-1}}{(-1+\eta \Phi)}, \\
x_{12 n+6} & =\frac{x_{0}}{-1+\zeta \Psi}, \\
x_{12 n+7} & =x_{6(2 n+2)-5}=x_{-5} .
\end{aligned}
$$

(i) When setting $\eta=1$ in Section 3.3.2, we get the result obtained in Theorem 4.1 in [4] for

$$
x_{n+1}=\frac{x_{n-5}}{-1+x_{n-1} x_{n-3} x_{n-5}},
$$

and their restriction coincides with our restriction (the initial conditions are arbitrary nonzero real numbers, $\Phi \neq 1$ and $\Psi \neq 1$ ).

(ii) When setting $\eta=-1$ in Section 3.3.2, we get the result obtained in Theorem 5.1 in [4] for

$$
x_{n+1}=\frac{x_{n-5}}{-1-x_{n-1} x_{n-3} x_{n-5}},
$$

and their restriction coincides with our restriction (the initial conditions are arbitrary nonzero real numbers, $\Phi \neq-1$ and $\Psi \neq-1)$.

\section{On the Difference Equation (5)}

Consider the fifth-order difference equations of the form (5), i.e.,

$$
u_{n+5}=\Omega=\frac{u_{n} u_{n+1} u_{n+2}}{u_{n+3} u_{n+4}\left(\lambda+\mu u_{n} u_{n+1} u_{n+2}\right)}
$$

Here, the procedure for finding the characteristics of (5) is similar as above and is as follows:

(i) Impose the symmetry condition (12) to (5)

(ii) Differentiate with respect to $u_{n}$ (keeping $\Omega$ fixed) and viewing $u_{n+3}$ as a function of $u_{n}, u_{n+1}, u_{n+2}$, and $\Omega$

(iii) Differentiate with respect to $u_{n}$ twice (keeping $u_{n+1}$ fixed)

(iv) Use the method of separation

After performing this series of operations, we obtain the following characteristics:

$$
\begin{aligned}
Q_{5}\left(n, u_{n}\right) & =\beta^{n} u_{n}, \\
Q_{6} & =\bar{\beta}^{n} u_{n},
\end{aligned}
$$

where $\beta=\exp (-2 \pi i / 3)$. Thus, we obtain two characteristics with corresponding generators given by

$$
\begin{aligned}
X_{5}= & \bar{\beta}^{n} u_{n} \partial u_{n}+\bar{\beta}^{n+1} u_{n+1} \partial_{u_{n+1}}+\beta \bar{\beta}^{n} u_{n+2} \partial_{u_{n+2}} \\
& +\bar{\beta}^{n} u_{n+3} \partial_{u_{n+3}}+\bar{\beta}^{n+1} u_{n+4} \partial_{u_{n+4}}, \\
X_{6}= & \beta^{n} u_{n} \partial u_{n}+\beta^{n+1} u_{n+1} \partial_{u_{n+1}}+\bar{\beta} \beta^{n} u_{n+2} \partial_{u_{n+2}} \\
& +\beta^{n} u_{n+3} \partial_{u_{n+3}}+\beta^{n+1} u_{n+4} \partial_{u_{n+4} .}
\end{aligned}
$$

From the characteristic equations 
$\frac{d u_{n}}{\bar{\beta}^{n} u_{n}}=\frac{d u_{n+1}}{\bar{\beta}^{n+1} u_{n+1}}=\frac{d u_{n+2}}{\bar{\beta}^{n+2} u_{n+2}}=\frac{d u_{n+3}}{\bar{\beta}^{n+3} u_{n+3}}=\frac{d u_{n+4}}{\bar{\beta}^{n+4} u_{n+4}}\left(=\frac{V_{n}}{0}\right)$,

we obtain the invariants $c_{1}=u_{n+1}^{\beta} / u_{n}, c_{2}=u_{n+2}^{\bar{\beta}} / u_{n}, c_{3}=$ $u_{n} u_{n+3}, c_{4}=u_{n+4}^{\beta} / u_{n}$, and $c_{5}=V_{n}$. We readily notice that

$$
S^{3}\left(\frac{d u_{n}}{\bar{\beta}^{n} u_{n}}=\frac{d u_{n+1}}{\bar{\beta}^{n+1} u_{n+1}}\right)=\frac{d u_{n+3}}{\bar{\beta}^{n+3} u_{n+3}}=\frac{d u_{n+4}}{\bar{\beta}^{n+4} u_{n+4}},
$$

and we choose $V_{n}=c_{1}^{\bar{\beta}} c_{2}^{\beta}$, i.e.,

$$
V_{n}=u_{n} u_{n+1} u_{n+2} \text {. }
$$

By shifting (49) thrice, we get

$$
V_{n+3}=\frac{V_{n}}{\lambda+\mu V_{n}}
$$

whose solution is given by

$$
V_{n}= \begin{cases}\left(\frac{\mu\left[\left((-1)^{2 / 3}-1\right) n-(-1)^{1 / 3}+1+\beta\right]}{3\left((-1)^{2 / 3}-1\right)}+c_{6}+\bar{\beta}^{n} c_{7}+\beta^{n} c_{8}\right)^{-1}, & \text { if } \lambda=1, \\ \left(c_{10} \lambda^{n / 3}+\left[(-1)^{2 / 3} \lambda^{1 / 3}\right]^{n} c_{8}+\left[-(-1)^{1 / 3} \lambda^{1 / 3}\right]^{n} c_{9}+\frac{\mu}{1-\lambda}\right)^{-1}, & \text { if } \lambda \neq 1 .\end{cases}
$$

The constants $c_{i}, i=6, \ldots, 10$, can be obtained from the following equations:

$$
\begin{aligned}
c_{6}+c_{7}+c_{8} & =\frac{1}{u_{0} u_{1} u_{2}}-\frac{\mu\left[-(-1)^{1 / 3}+1+\beta\right]}{3\left((-1)^{2 / 3}-1\right)}, \\
c_{6}+\bar{\beta} c_{7}+\beta c_{8} & =\frac{1}{u_{1} u_{2} u_{3}}-\frac{\mu\left[\beta-(-1)^{1 / 3}+(-1)^{2 / 3}\right]}{3\left((-1)^{2 / 3}-1\right)}, \\
c_{6}+\beta c_{7}+\bar{\beta} c_{8} & =\frac{1}{u_{2} u_{3} u_{4}}-\frac{\mu\left[-1+2(-1)^{2 / 3}-(-1)^{1 / 3}+\beta\right]}{3\left((-1)^{2 / 3}-1\right)}, \\
c_{10}+c_{8}+c_{9} & =\frac{1}{u_{0} u_{1} u_{2}}-\frac{\mu}{1-\lambda}, \\
\left.\lambda^{1 / 3} c_{10}+\left[(-1)^{2 / 3} \lambda^{1 / 3}\right] c_{8}\right]+\left[-(-1)^{1 / 3} \lambda^{1 / 3}\right] c_{9} & =\frac{1}{u_{1} u_{2} u_{3}}-\frac{\mu}{1-\lambda}, \\
\lambda^{2 / 3} c_{10}+\left[(-1)^{2 / 3} \lambda^{1 / 3}\right]^{2} c_{8}+\left[-(-1)^{1 / 3} \lambda^{1 / 3}\right]^{2} c_{9} & =\frac{1}{u_{2} u_{3} u_{4}}-\frac{\mu}{1-\lambda} .
\end{aligned}
$$

Thanks to (49), we can express $u_{n}$ in terms of $V_{n}$ as follows:

$$
u_{n}=\exp \left(\beta^{n} c_{11}+\bar{\beta}^{n} c_{12}-\frac{2}{\sqrt{3}}\left[\sum_{k=0}^{n-1} \operatorname{Im}(\gamma(n, k)) \ln V_{k}\right]\right),
$$

where $V_{k}$ is given in (51) with $\gamma(n, k)=\beta^{n} \bar{\beta}^{k+1}$. The constants $c_{11}$ and $c_{12}$ must satisfy

$$
\begin{aligned}
c_{11}+c_{12} & =\ln u_{0}, \\
\beta c_{11}+\bar{\beta} c_{12} & =\ln u_{1} .
\end{aligned}
$$

Equations in (53a) and (53b) give the solutions of (5) in a unified manner.

For the sake of clarification, we split solutions (53a) to realize the solutions in the existing literature. Using (53a) and (53b), we have

$$
u_{6 n}=u_{0} \prod_{s=1}^{2 n} \frac{V_{3 s-2}}{V_{3 s-3}}
$$

Using the same approach, we show that

$$
u_{6 n+i}=u_{i} \prod_{s=1}^{2 n} \frac{V_{3(s-1)+i+1}}{V_{3(s-1)+i}}, \quad i=0, \ldots, 5 .
$$


4.1. The Case of $\lambda=1$. Equation (5) becomes

$$
u_{n+5}=\frac{u_{n} u_{n+1} u_{n+2}}{u_{n+3} u_{n+4}\left(1+\mu u_{n} u_{n+1} u_{n+2}\right)},
$$

and we said earlier that the solution of (50), in this case, is (51), i.e.,

$V_{n}=\left(\frac{\mu\left[\left((-1)^{2 / 3}-1\right) n-(-1)^{1 / 3}+1+\beta\right]}{3\left((-1)^{2 / 3}-1\right)}+c_{6}+\bar{\beta}^{n} c_{7}+\beta^{n} c_{8}\right)^{-1}$.

We have

$$
V_{3 s}=\left(\frac{\mu\left[\left((-1)^{2 / 3}-1\right)(3 s)-(-1)^{1 / 3}+1+\beta\right]}{3\left((-1)^{2 / 3}-1\right)}+c_{6}+c_{7}+c_{8}\right)^{-1},
$$

and using (52a) in (58), we get

$$
V_{3 s}=\frac{u_{0} u_{1} u_{2}}{1+\mu s u_{0} u_{1} u_{2}} \text {. }
$$

Using the same approach, we show that

$$
\begin{gathered}
V_{3 s}=\frac{u_{0} u_{1} u_{2}}{1+\mu s u_{0} u_{1} u_{2}}, \\
V_{3 s+1}=\frac{u_{1} u_{2} u_{3}}{1+\mu s u_{1} u_{2} u_{3}}, \\
V_{3 s+2}=\frac{u_{2} u_{3} u_{4}}{1+\mu s u_{2} u_{3} u_{4}} .
\end{gathered}
$$

Let $a=x_{-4}, b=x_{-3}, c=x_{-2}$, and $d=x_{-1}$ and $A=u_{0}$, $B=u_{1}, C=u_{2}, D=u_{3}$, and $E=u_{4}$. Using (60) in (55), we obtain the solution of (56) as follows:

$$
\begin{aligned}
& u_{6 n}= \frac{D^{2 n}}{A^{2 n-1}} \prod_{s=1}^{2 n-1} \frac{1+\mu s A B C}{1+\mu s B C D}, \\
& u_{6 n+1}= \frac{E^{2 n}}{B^{2 n-1}} \prod_{s=1}^{2 n-1} \frac{1+\mu s B C D}{1+\mu s C D E}, \\
& u_{6 n+2}= \frac{C A^{2 n} B^{2 n}}{D^{2 n} E^{2 n}} \prod_{s=0}^{2 n-1} \frac{1+\mu s C D E}{1+\mu(s+1) A B C}, \\
& u_{6 n+3}=\frac{D^{2 n+1}}{A^{2 n}} \prod_{s=1}^{2 n} \frac{1+\mu s A B C}{1+\mu s B C D}, \\
& u_{6 n+4}=\frac{E^{2 n+1}}{B^{2 n}} \prod_{s=0}^{2 n} \frac{1+\mu s B C D}{1+\mu s C D E}, \\
& u_{6 n+5}=\frac{C(A B)^{2 n+1}}{(D E)^{2 n+1}} \prod_{s=0}^{2 n} \frac{1+\mu s C D E}{1+\mu(s+1) A B C},
\end{aligned}
$$

whenever the denominators do not vanish.

4.2. The Case of $\lambda \neq 1$. In this case, as we found earlier, the solution of (50) is given by (51), i.e.,

$$
V_{n}=\left(c_{10} \lambda^{n / 3}+\left[(-1)^{2 / 3} \lambda^{1 / 3}\right]^{n} c_{8}+\left[-(-1)^{1 / 3} \lambda^{1 / 3}\right]^{n} c_{9}+\frac{\mu}{1-\lambda}\right)^{-1} .
$$

Using this, we get

$$
V_{3 s}=\left(c_{10} \lambda^{s}+c_{8} \lambda^{s}+c_{9} \lambda^{s}+\frac{\mu}{1-\lambda}\right)^{-1}
$$

and using (52d) in (63), we find

$$
V_{3 s}=\frac{A B C}{\lambda^{s}+\mu A B C\left(\left(1-\lambda^{s}\right) /(1-\lambda)\right)} .
$$

Using the same approach, we show that

$$
V_{3 s+i}=\frac{u_{i} u_{i+1} u_{i+2}}{\lambda^{s}+\mu u_{i} u_{i+1} u_{i+2}\left(\left(1-\lambda^{s}\right) /(1-\lambda)\right)}, \quad i=0,1,2 .
$$

Using (65) in (55), we obtain the solution of (5) as follows:

$$
\begin{gathered}
u_{6 n}=\frac{D^{2 n}}{A^{2 n-1}} \prod_{s=2}^{2 n} \frac{\lambda^{s-1}+\mu \Delta_{0} \sum_{j=0}^{s-2} \lambda^{j}}{\lambda^{s-1}+\mu \Delta_{1} \sum_{j=0}^{s-2} \lambda^{j}}, \\
u_{6 n+1}=\frac{E^{2 n}}{B^{2 n-1}} \prod_{s=2}^{2 n} \frac{\lambda^{s-1}+\mu \Delta_{1} \sum_{j=0}^{s-2} \lambda^{j}}{\lambda^{s-1}+\mu \Delta_{2} \sum_{j=0}^{s-2} \lambda^{j}}, \\
u_{6 n+2}=C \frac{\Delta_{0}^{2 n}}{\Delta_{1}^{2 n}} \frac{\prod_{s=2}^{2 n}\left(\lambda^{s-1}+\mu \Delta_{2} \sum_{j=0}^{s-2} \lambda^{j}\right)}{\prod_{s=1}^{2 n}\left(\lambda^{s}+\mu \Delta_{0} \sum_{j=0}^{s-1} \lambda^{j}\right)}, \\
u_{6 n+3}=\frac{D^{2 n+1}}{A^{2 n}} \prod_{s=2}^{2 n+1} \frac{\lambda^{s-1}+\mu \Delta_{0} \sum_{j=0}^{s-2} \lambda^{j}}{\lambda^{s-1}+\mu \Delta_{1} \sum_{j=0}^{s-2} \lambda^{j}}, \\
u_{6 n+4}=E \frac{\Delta_{2}^{2 n}}{\Delta_{1}^{2 n}} \prod_{s=2}^{2 n+1} \frac{\lambda^{s-1}+\mu \Delta_{1} \sum_{j=0}^{s-2} \lambda^{j}}{\lambda^{s-1}+\mu \Delta_{2} \sum_{j=0}^{s-2} \lambda^{j}}, \\
u_{6 n+5}=C \frac{\Delta_{0}^{2 n+1}}{\Delta_{1}^{2 n+1}} \frac{\prod_{s=2}^{2 n+1}\left(\lambda^{s-1}+\mu \Delta_{2} \sum_{j=0}^{s-2} \lambda^{j}\right)}{\prod_{s=1}^{2 n+1}\left(\lambda^{s}+\mu \Delta_{0} \sum_{j=0}^{s-1} \lambda^{j}\right)},
\end{gathered}
$$

where $\Delta_{i}=u_{i} u_{i+1} u_{i+2}$. Equations in Section 4.2 give the exact solution of (5) for any real values of $\lambda$ and $\mu$ provided that the denominators do not vanish.

Recall that we acted the shift operator on (4) to get (5). Hence, the solutions of (4) are obtained, using Section 4.2, as follows: 


$$
\begin{aligned}
x_{6 n-4}= & \frac{d^{2 n}}{a^{2 n-1}} \prod_{s=2}^{2 n} \frac{\lambda^{s-1}+\mu a b c \sum_{j=0}^{s-2} \lambda^{j}}{\lambda^{s-1}+\mu b c d \sum_{j=0}^{s-2} \lambda^{j}}, \\
x_{6 n-3} & =\frac{e^{2 n}}{b^{2 n-1}} \prod_{s=2}^{2 n} \frac{\lambda^{s-1}+\mu b c d \sum_{j=0}^{s-2} \lambda^{j}}{\lambda^{s-1}+\mu c d e \sum_{j=0}^{s-2} \lambda^{j}}, \\
x_{6 n-2} & =\frac{c(a b)^{2 n}}{(d e)^{2 n}} \frac{\prod_{s=2}^{2 n}\left(\lambda^{s-1}+\mu c d e \sum_{j=0}^{s-2} \lambda^{j}\right)}{\prod_{s=1}^{2 n}\left(\lambda^{s}+\mu a b c \sum_{j=0}^{s-1} \lambda^{j}\right)}, \\
x_{6 n-1} & \frac{d^{2 n+1}}{a^{2 n}} \prod_{s=2}^{2 n+1} \frac{\lambda^{s-1}+\mu a b c \sum_{j=0}^{s-2} \lambda^{j}}{\lambda^{s-1}+\mu b c d \sum_{j=0}^{s-2} \lambda^{j}}, \\
x_{6 n}= & \frac{e^{2 n+1}}{b^{2 n}} \prod_{s=2}^{2 n+1} \frac{\lambda^{s-1}+\mu b c d \sum_{j=0}^{s-2} \lambda^{j}}{\lambda^{s-1}+\mu c d e \sum_{j=0}^{s-2} \lambda^{j}}, \\
x_{6 n+1} & =\frac{c(a b)^{2 n+1}}{(d e)^{2 n+1}} \frac{\prod_{s=2}^{2 n+1}\left[\lambda^{s-1}+\mu c d e \sum_{j=0}^{s-2} \lambda^{j}\right]}{\prod_{s=1}^{2 n+1}\left(\lambda^{s}+\mu a b c \sum_{j=0}^{s-1} \lambda^{j}\right)},
\end{aligned}
$$

for any real values of $\lambda$ and $\mu$ as long as the denominators do not vanish.

(i) When $\lambda=1$ and $\mu=1$, equations in Section 4.2 yield the results obtained by Yasin Yazlik in Theorem 5 in [9] for

$$
x_{n+1}=\frac{x_{n-2} x_{n-3} x_{n-4}}{x_{n} x_{n-1}\left(1+x_{n-2} x_{n-3} x_{n-4}\right)}, \quad n=0,1,2, \ldots,
$$

where $a, b, c, c, d$, and $e$ are positive real numbers.

(ii) When $\lambda=1$ and $\mu=-1$, equations in Section 4.2 yield the results obtained by Yasin Yazlik in Theorem 9 in [9] for

$$
x_{n+1}=\frac{x_{n-2} x_{n-3} x_{n-4}}{x_{n} x_{n-1}\left(1-x_{n-2} x_{n-3} x_{n-4}\right)}, \quad n=0,1,2, \ldots,
$$

where $a, b, c, c, d$, and $e$ are positive real numbers with $a b c \neq 1$ and $c$ de $\neq 1$.

Note. There should not be a minus sign right after the expression of $x_{3 n-2}$ in Theorem 9 in [9].

(i) When $\lambda=-1$ and $\mu=1$, equations in Section 4.2 yield the results obtained by Yasin Yazlik in Theorem 7 in [9] for

$$
x_{n+1}=\frac{x_{n-2} x_{n-3} x_{n-4}}{x_{n} x_{n-1}\left(-1+x_{n-2} x_{n-3} x_{n-4}\right)}, \quad n=0,1,2, \ldots
$$

where $a, b, c, d$, and $e$ are nonzero real numbers with $a b c \neq 1, b c d \neq 1$, and $c d e \neq 1$. (ii) When $\lambda=-1$ and $\mu=-1$, equations in Section 4.2 yield the results obtained by Yasin Yazlik in Theorem 11 in [9] for

$$
x_{n+1}=\frac{x_{n-2} x_{n-3} x_{n-4}}{x_{n} x_{n-1}\left(-1-x_{n-2} x_{n-3} x_{n-4}\right)}, \quad n=0,1,2, \ldots,
$$

where $a, b, c, c, d$, and $e$ are nonzero real numbers with $a b c \neq-1, b c d \neq-1$, and $c d e \neq-1$.

Note. There should be a minus sign right after the expression of $x_{6 n+1}$ in Theorem 11 in [9].

\section{Conclusion}

In this paper, we have obtained nontrivial symmetries of some rational ordinary difference equations and their exact solutions were obtained. Most importantly, we note that (21) gives a clear idea, without making any lucky guesses, for the most convenient choice of the invariant of (3).

\section{Data Availability}

No data were used to support this study.

\section{Conflicts of Interest}

The authors declare that there are no conflicts of interest regarding the publication of this paper.

\section{References}

[1] S. Lie, "Classification und integration von gewöhnlichen differentialgleichungen zwischenxy, die eine gruppe von transformationen gestatten," Mathematische Annalen, vol. 32, no. 2, pp. 213-281, 1888.

[2] E. Noether, "Invariante variationsprobleme," MathematischPhysikalische Klasse, vol. 2, pp. 235-257, 1918.

[3] P. E. Hydon, Difference Equations by Differential Equation Methods, Cambridge University Press, Cambridge, UK, 2014.

[4] E. M. Elsayed, "Dynamics of a rational recursive sequence," International Journal of Differential Equations, vol. 4, no. 2, pp. 185-200, 2009.

[5] M. Folly-Gbetoula, "Symmetry, reductions and exact solutions of difference equations $u_{n+2}=a u_{n} /\left(1+b u_{n} u_{n+1}\right)$.," Journal of Difference Equations and Applications, vol. 23, no. 6, pp. 1017-1024, 2017.

[6] M. Folly-Gbetoula and A. H. Kara, "Symmetries, conservation laws, and integrability of difference equations," Advances in Difference Equations, vol. 2014, no. 1, p. 224, 2014.

[7] S. Steviç, J. Diblik, B. Iricanin, and Z. Smarda, "On a fifth-order difference equation," Journal of Computational Analysis and Applications, vol. 20, no. 7, pp. 1214-1227, 2016.

[8] N. Joshi and P. J. Vassiliou, "The existence of Lie symmetries for first-order analytic discrete dynamical systems," Journal of Mathematical Analysis and Applications, vol. 195, no. 3, pp. 872-887, 1995.

[9] Y. Yazlik, "On the solutions and behavior of rational difference equations," Journal of Computational Analysis and Applications, vol. 17, no. 3, pp. 584-594, 2014. 


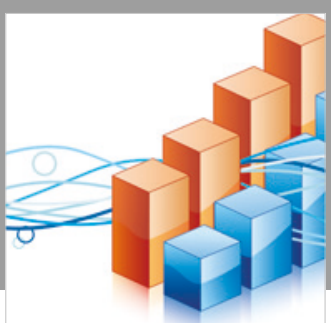

Advances in

Operations Research

\section{-n-m}
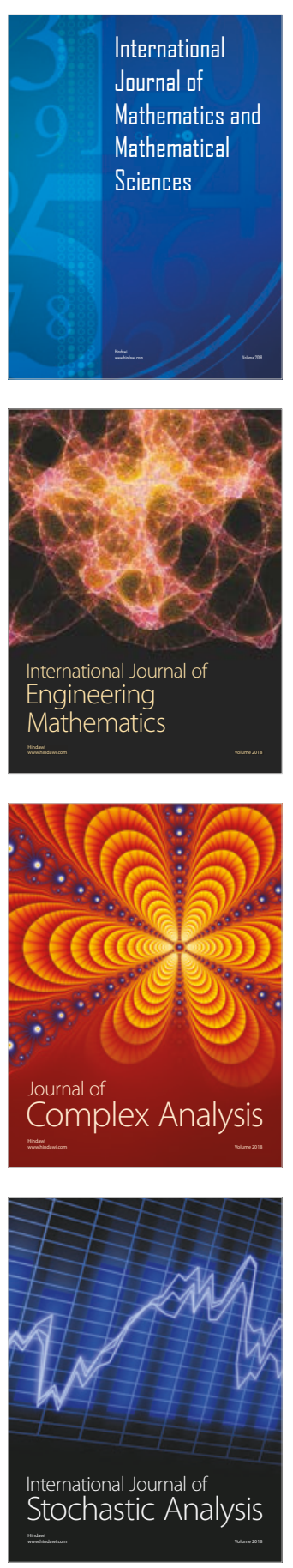
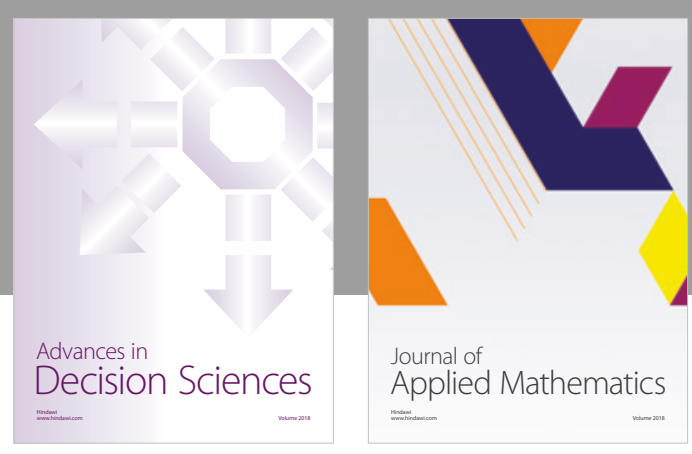

Journal of

Applied Mathematics
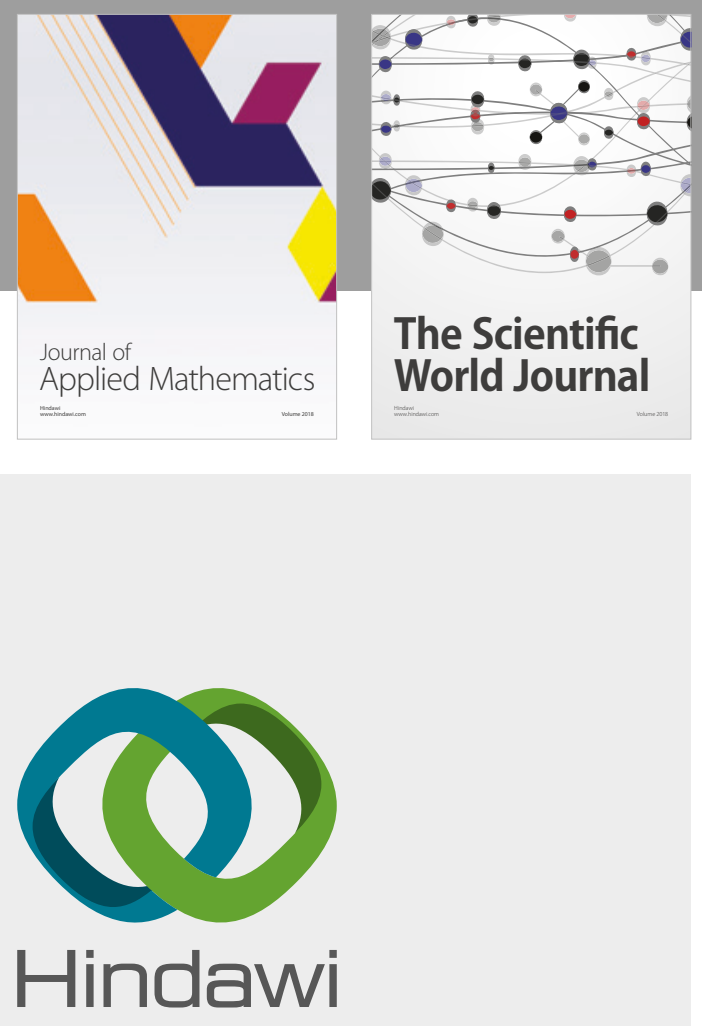

Submit your manuscripts at

www.hindawi.com

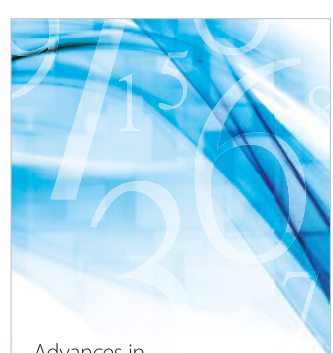

Advances in
Numerical Analysis
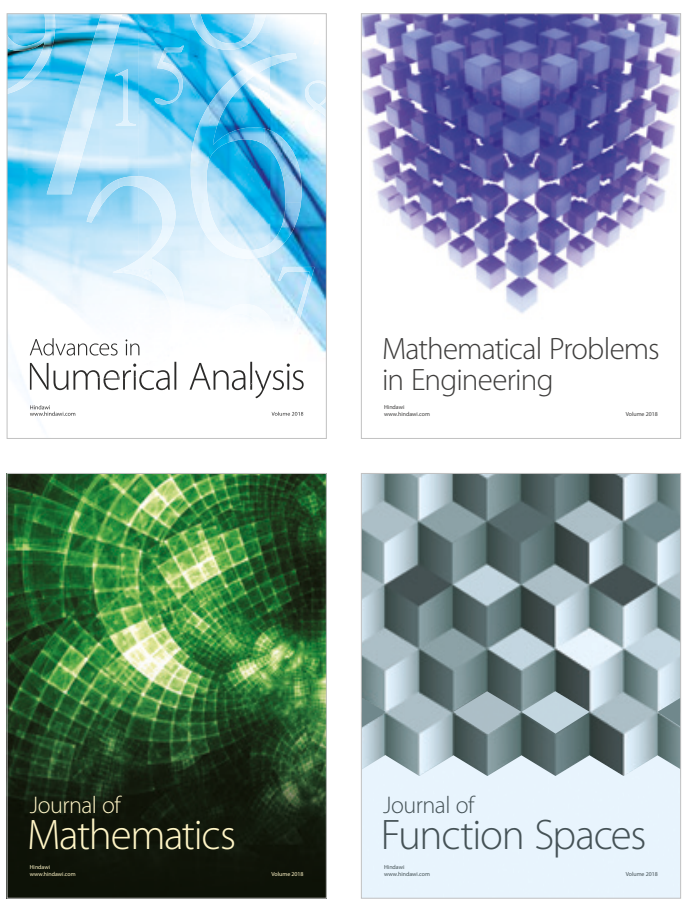

Mathematical Problems in Engineering

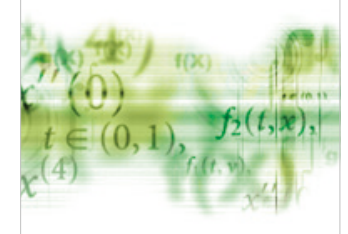

International Journal of

Differential Equations

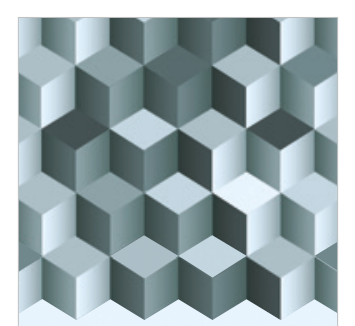

Journal of

Function Spaces

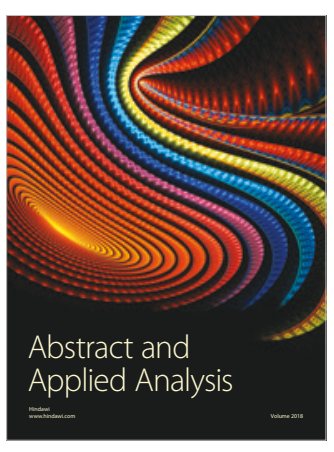

The Scientific

World Journal

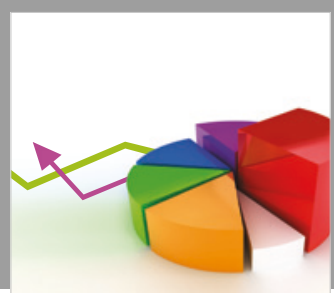

Journal of

Probability and Statistics
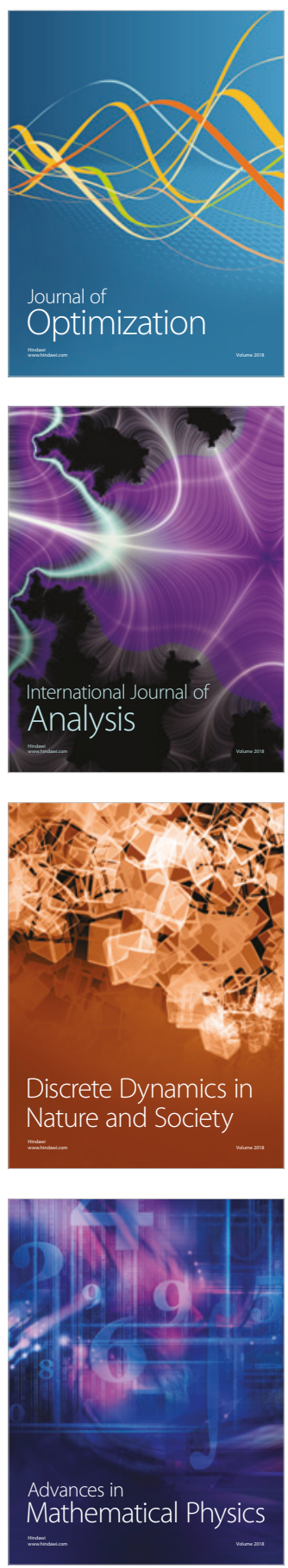